\title{
EFEITO DO FRIO E DO DESPONTE NA BROTAÇÃO DE GEMAS EM PESSEGUEIRO ${ }^{1}$
}

\author{
FERNANDO JOSÉ HAWERROTH², JOSÉ LUIZ PETRI ${ }^{3}$, GABRIEL BERENHAUSER LEITE4, \\ FLAVIO GILBERTO HERTER ${ }^{5}$, ANDERSON CARLOS MARAFON ${ }^{6}$
}

RESUMO -O objetivo do trabalho foi avaliar a resposta de três cultivares de pessegueiro quanto à brotação de gemas, em ramos com ou sem desponte, quando submetidas a diferentes períodos de exposição ao frio. Ramos de ano das cultivares Coral, Eragil e Rubidoux foram submetidas ao frio complementar (0; 312; 624 e 1.248 unidades de frio). Posteriormente, metade dos ramos foram mantidos inteiros e nos demais efetuou-se o desponte, com a retirada da gema apical. Os ramos foram mantidos em câmara de crescimento a $25^{\circ} \mathrm{C}$, sendo avaliado o percentual de brotação de gemas vegetativas e floríferas aos 15 e 30 dias após início da exposição a $25^{\circ} \mathrm{C}$. O desponte incrementou a brotação de gemas vegetativas em todas as cultivares avaliadas. O efeito do desponte sobre a brotação das gemas vegetativas foi variável conforme as unidades de frio aos quais os ramos foram expostos, sendo evidenciado maior efeito do desponte nos tratamentos com maior exposição ao frio.

Termos para indexação: dominância apical, dormência, Prunus persica (L.) Batsch, requerimento em frio.

\section{CHILLING AND TIPPING OFF PRUNING EFFECT ON BUDBREAK IN PEACH TREES}

\begin{abstract}
The objective of this work was to evaluate the behavior of three peach cultivars in relation to budbreak in branches with or without tipping off pruning submitted to different exposition cold periods. One-year-old branches of Coral, Eragil and Rubidoux peach trees cultivars were submitted to chilling supplement (0, 312, 624 and 1248 cold units). After chilling treatments, half of branches were maintained intact and others were tipped off pruning through terminal bud remove. After this, the branches were kept in growth chamber at $25^{\circ} \mathrm{C}$, and it was evaluated the percentage of budbreak in flower and vegetative buds at 15 and 30 days after the beginning of the exposure at $25^{\circ} \mathrm{C}$. Tipping off pruning increased budbreak in vegetative buds in all cultivars studied. The effect of tipping off pruning on budbreak in vegetative buds was variable depending on chilling intensity that the branches were submitted, being evidenced bigger effect of tipping off pruning in higher chilling exposure.
\end{abstract}

Index terms: apical dominance, dormancy, Prunus persica (L.) Batsch, chilling requirement.

\section{INTRODUÇÃO}

O pessegueiro (Prunus persica (L.) Batsch.) é originário de regiões tipicamente frias, caracterizadas pela alta quantidade de frio acumulado durante o período hibernal. Nestas condições, a dormência foi um importante mecanismo adaptativo adquirido pelas frutíferas de clima temperado, visando à sua sobrevivência em condições inadequadas ao seu desenvolvimento, como baixas temperaturas. Este fenômeno é caracterizado pela aquisição da resistência ao frio e controle do crescimento (Leite, 2005), onde as atividades metabólicas das plantas continuam, embora em intensidade reduzida (Petri et al., 2006).

A baixa temperatura ambiente, durante 0 período de dormência é, reconhecidamente, o principal agente natural envolvido na indução da brotação das plantas (Petri, 1986; Erez, 2000), sendo que a maioria das frutíferas temperadas necessitam ser submetidas a um período de baixas temperaturas

\footnotetext{
1(Trabalho 046-08). Recebido em: 27-02-2008. Aceito para publicação em: 03-03-2009.

${ }^{2}$ Eng. Agr., MSc., Bolsista de Doutorado do CNPq, Doutorando em Agronomia - Concentração em Fruticultura de Clima Temperado, Universidade Federal de Pelotas, C.P. 354, 96010-900, Pelotas-RS. Email: fjhawerroth@gmail.com ${ }^{3}$ Eng. Agr., MSc., Epagri - Estação Experimental de Caçador, C.P. 591, 89500-000, Caçador-SC. E-mail: petri@epagri.rctsc.br

${ }^{4}$ Eng. Agr., Dr., Epagri - Estação Experimental de Caçador, C.P. 591, 89500-000, Caçador-SC. E-mail: gabriel@epagri.rctsc.br

${ }^{5}$ Eng. Agr., Dr., Embrapa Clima Temperado, C.P. 403, 96001-970, Pelotas-RS. E-mail: herter@cpact.embrapa.br

${ }^{6}$ Eng. Agr., Dr., Universidade Federal de Pelotas, C.P. 354, 96010-900, Pelotas-RS. Email: anderson_marafon@ufpel.edu.br
} 
para posterior retomada de crescimento na primavera (Zelleke \& Kliewer, 1989). Em condições normais de clima temperado, após o frio recebido durante o inverno, espécies frutíferas decíduas retomam seu ciclo vegetativo e reprodutivo normalmente (Leite, 2005), apresentando abundante brotação de gemas.

Com a expansão da fruticultura de clima temperado para regiões de inverno ameno e até mesmo subtropicais, onde o frio é insuficiente para satisfazer as necessidades fisiológicas da planta para sair da dormência (Botelho et al., 2006), problemas relacionados à brotação e floração deficientes manifestam-se com maior frequência, repercutindo em redução da produtividade e da qualidade dos frutos produzidos.

A utilização de cultivares com requerimento em frio compatível com as condições climáticas de uma determinada região de cultivo é fundamental para o sucesso na produção comercial de fruteiras decíduas em regiões de clima ameno. Neste sentido, a quantificação do frio é importante para determinar o requerimento em frio de cada cultivar e para definir a quantidade de frio disponível em um local específico (Erez, 2000).

Além do atendimento do requerimento em frio de uma cultivar, outros fatores estão envolvidos com o desencadeamento do processo de brotação, como a necessidade de calor (Citadin, 1999), o uso de substâncias químicas (Petri et al., 2006) ou mesmo práticas culturais como a desfolha e a poda (Tromp, 2005). Dada a relevância da interação entre esses fatores na dinâmica da dormência, estudos visando a seu entendimento são de grande importância para desenvolver e adequar práticas culturais com o intuito de minimizar os problemas decorrentes do insuficiente acúmulo de frio durante o período hibernal.

Entre os métodos disponíveis para estudar a dormência em fruteiras de clima temperado, destacase o método biológico. Este método baseia-se na utilização de ramos produtivos de uma espécie ou cultivar em específico, expondo-os a diferentes tratamentos de frio, e posteriormente submetendoos ao calor para estimular a brotação de gemas, quantificando-a ao longo do tempo. Este método tem sido utilizado para várias espécies de clima temperado, como a macieira (Herter et al., 1992; Putti et al., 2003), a pereira (Bianchi et al., 2000) e o pessegueiro (Citadin, 1999).

O presente trabalho teve por objetivo avaliar, através do método biológico, a resposta de três cultivares de pessegueiro quanto à brotação de gemas, em ramos de ano, em resposta à exposição ao frio e à poda de desponte.

\section{MATERIAL E MÉTODOS}

O experimento foi conduzido nas dependências da Epagri/Estação Experimental de Caçador-SC. Ramos de pessegueiro de crescimento do ano, com 25 a $30 \mathrm{~cm}$ de comprimento, das cultivares Coral, Eragil e Rubidoux, foram coletados de plantas com 6 anos de idade, em pomar comercial localizado em Caçador-SC (latitude 2642’32" sul, longitude $51^{\circ} 00^{\prime} 50^{\prime \prime}$ oeste e altitude de 960 metros). As cultivares utilizadas apresentam distintas exigências em frio, onde, segundo Biasi et al. (2004), as cultivares Coral, Eragil e Rubidoux apresentam requerimento de 350; 550 e 600 horas de frio abaixo de $7,2^{\circ} \mathrm{C}$, respectivamente. A coleta dos ramos foi realizada em 24-06-2006, quando já haviam acumulado 43 horas de frio abaixo de $7,2^{\circ} \mathrm{C}$ ou 290 unidades de frio, segundo modelo de Utah (Richardson et al., 1974). Os ramos foram separados, formando quatro lotes com 20 ramos para cada cultivar, os quais foram submetidos a diferentes períodos de exposição ao frio, para o qual se utilizou uma câmara frigorífica a $3^{\circ} \mathrm{C}\left( \pm 1^{\circ} \mathrm{C}\right)$. Adotando o modelo de acumulação de unidades de frio proposto por Richardson et al. (1974), desenvolvido para pessegueiros, considerou-se uma unidade de frio (UF) a cada hora de permanência dos ramos em câmara frigorífica a $3^{\circ} \mathrm{C}$.

Os ramos foram submetidos às seguintes unidades de frio complementar: 0; 312; 624 e 1.248 UF. Depois de atendidos os períodos de exposição respectivos a cada tratamento, cada lote foi dividido em duas partes, sendo dez ramos mantidos inteiros e, nos restantes, foi realizada a poda da gema apical. O corte para a retirada da gema apical foi efetuado a $1 \mathrm{~cm}$ abaixo do ponto de inserção da mesma no ramo. Esses ramos foram colocados em bandejas com espuma fenólica e mantidos em câmara de crescimento a $25^{\circ} \mathrm{C} \pm 1^{\circ} \mathrm{C}$, com umidade relativa média de $85 \%$ e fotoperíodo de 12 horas.

O delineamento experimental utilizado foi o inteiramente casualizado, com cinco repetições compostas de dois ramos. Adotou-se o arranjo fatorial ( 4 × 3 x 2), obtido da combinação de 4 níveis do fator exposição ao frio ( 0 ; 312; 624 e 1.248 UF), 3 níveis do fator cultivar (Coral, Eragil e Rubidoux) e 2 níveis do fator desponte (ramos despontados e não despontados).

As avaliações consistiram na determinação dos percentuais de brotação de gemas floríferas e de gemas vegetativas, aos 15 e 30 dias após a manutenção dos ramos em câmara de crescimento a $25^{\circ} \mathrm{C}$. As gemas vegetativas e floríferas foram consideradas brotadas quando se encontravam a 
partir do estágio de ponta verde e botão rosado, respectivamente.

As variáveis avaliadas foram transformadas através da expressão $\sqrt{(x+1)}$ a fim de atender às pressuposições em homogeneidade de variância (Banzatto \& Kronka, 1995), para então serem submetidas à análise de variância. As variáveis cujos resultados revelaram significância a 5\% de probabilidade de erro, tiveram as médias comparadas pelo teste de Tukey, a 5\% de significância, para os fatores qualitativos cultivar e desponte, e para o fator quantitativo exposição ao frio, foi efetuado o ajuste de equações de regressão polinomial.

\section{RESULTADOS E DISCUSSÃO}

O efeito principal dos fatores intensidade de frio, cultivar e desponte foram significativos quanto ao percentual de gemas vegetativas brotadas após 15 e 30 dias a $25^{\circ} \mathrm{C}$, assim como a interação entre os fatores intensidade de frio e desponte. A interação entre o fator intensidade de frio e cultivar foi significativo apenas para a variável brotação de gemas vegetativas aos 15 dias a $25^{\circ} \mathrm{C}$ (Tabela 1).

A poda de desponte proporcionou aumento da brotação de gemas vegetativas em todas as cultivares estudadas, sendo visualizado seu efeito tanto na avaliação aos 15 dias como aos 30 dias após a exposição ao calor a $25^{\circ} \mathrm{C}$ (Tabela 2). De acordo com Mondin (2005), a poda de desponte estimula o desenvolvimento das gemas situadas abaixo do local onde o corte foi efetuado, resultado também observado neste experimento. O efeito da poda da gema apical sobre a maximização da brotação pode ser observado na cultivar Eragil, onde o desponte proporcionou aumento de 2,73 e 2,60 vezes no percentual de gemas vegetativas brotadas aos 15 e 30 dias em ambiente a $25^{\circ} \mathrm{C}$, respectivamente. Essa resposta pode ser creditada à eliminação da paradormência, neste caso, caracterizada pela dominância apical, que inibe a brotação das gemas laterais (Cook \& Jacobs, 1999; Leite, 2004).

Segundo Taiz \& Zeiger (2004), na maioria das plantas superiores, o desenvolvimento das gemas apicais inibe o crescimento e o desenvolvimento das gemas axilares situadas abaixo destas, num processo característico de dominância apical. Com a retirada da gema apical, a dominância apical exercida sobre as gemas laterais é eliminada, ocorrendo, segundo Barrit (1998), melhor distribuição da seiva, favorecendo a brotação de gemas axilares.

O efeito do desponte sobre a brotação de gemas vegetativas foi diferenciado entre os períodos de exposição ao frio estudados, como pode ser observado na Figura 1. Nos menores períodos de exposição ao frio (0 UF e 312 UF), o efeito do desponte no aumento da brotação de gemas foi menos pronunciado em relação às maiores exposições ao frio. Tal comportamento pode estar associado ao fato de que grande parte das gemas dos ramos submetidos a 0 UF e 312 UF se encontravam, possivelmente, no estado de endodormência. Segundo Lang (1987), a endodormência é caracterizada pela paralisação do desenvolvimento da gema, não sendo manifestada a brotação das gemas mesmo quando as condições ambientes sejam adequadas para tal. Ao atingir este estado, o frio é o único fator capaz de tornar a gema fisiologicamente apta a brotar (Petri \& Herter, 2004).

A partir das equações de regressão polinomial (Figura 1), foi verificado o mesmo percentual de gemas vegetativas brotadas em ramos despontados e não despontados com 104 UF. O aumento da exposição ao frio, a partir deste ponto, maximizou as diferenças na brotação entre ramos despontados e não despontados, sendo a máxima diferença obtida com 1.120 UF.

As cultivares utilizadas apresentaram respostas diferenciadas na brotação de gemas vegetativas mediante os períodos de exposição ao frio estudados (Figura 2). Aos 15 dias, a $25^{\circ} \mathrm{C}$, a cultivar Coral apresentou o maior aumento na brotação de gemas vegetativas frente ao maior número de horas de exposição dos ramos ao frio, atingindo máxima brotação em 778 UF. Igualmente à cultivar Coral, Rubidoux apresentou resposta quadrática ao frio, porém o máximo percentual de brotação de gemas vegetativas foi inferior ao da cultivar Coral, sendo atingido com 1.143 UF. A cultivar Eragil respondeu linearmente ao frio, mostrando apresentar incremento na brotação de gemas vegetativas com períodos de exposição ao frio superiores aos utilizados neste estudo.

Aos 30 dias a $25^{\circ} \mathrm{C}$, o aumento da exposição ao frio proporcionou incremento no percentual de gemas vegetativas brotadas, embora a 326 e 275 UF este tenha sido inferior ao observado na intensidade 0 UF nas cultivares Coral e Eragil, respectivamente (Figura 2). Este comportamento, segundo Lang (1987) e Putti et al. (2003), pode estar relacionado ao fato de que, quando os ramos foram coletados, suas gemas estavam no estádio de paradormência e, com o aumento da exposição ao frio, entraram em endodormência. Como descrito por Lang (1987) e Petri \& Herter (2004), a ocorrência de frio, inicialmente, determina a fase de endodormência, e, após atingir este estado, o frio contribui para a saída desta fase, 
tornando as gemas aptas fisiologicamente a brotarem, o que explica o aumento significativo da brotação pela exposição dos ramos ao frio, superiores a 326 e 275 UF para as cultivares Coral e Eragil, respectivamente.

Aos 30 dias a $25^{\circ} \mathrm{C}$, não foram verificadas diferenças entre as cultivares Coral e Rubidoux, quando expostas a 312; 624 e 1248UF (Tabela 3). Segundo Biasi et al. (2004), as cultivares Coral e Rubidoux são contrastantes quanto à necessidade de frio, apresentando requerimento de 350 e 600 horas de frio abaixo de $7,2^{\circ} \mathrm{C}$, respectivamente. A inexistência de diferenças no nível de brotação entre as cultivares Coral e Rubidoux poderia ser esperada com 1.248 UF, visto que esta exposição ao frio é muito superior ao requerimento em frio das cultivares utilizadas. Dessa a forma, a avaliação realizada aos 30 dias a $25^{\circ} \mathrm{C}$ apresentou menor desempenho na separação das cultivares segundo requerimento em frio em relação à avaliação realizada aos 15 dias a $25^{\circ} \mathrm{C}$.

Durante a condução deste trabalho, observou-se unicamente brotação de gemas vegetativas, sendo o número de gemas floríferas brotadas praticamente desprezível em todas as intensidades de frio estudadas, motivo pelo qual não se efetuou a análise do percentual de brotação destas. Os ramos utilizados no experimento possuíam maior proporção de gemas floríferas do que de gemas vegetativas, visto que os ramos apresentavam a maior parte das gemas vegetativas na posição central, flanqueadas por duas gemas de flor, em um mesmo nó. Pelo fato de serem observadas brotação e floração abundantes na área onde se efetuou a coleta dos ramos, a ausência de brotação das gemas floríferas não está associada a problemas relacionados à qualidade dos ramos e ao manejo das plantas no pomar.
Quando expostos a 0 UF também não foi verificada brotação de gemas floríferas, excluindo a hipótese de possível necrose das gemas floríferas durante a submissão dos ramos aos tratamentos de frio na câmara fria. Em razão de ter ocorrido brotação de gemas vegetativas, o não suprimento da necessidade de frio das gemas floríferas pelas quantidades de frio estudadas não pode ser considerada a causa da não brotação de gemas floríferas, visto que, segundo Faust et al. (1995), gemas floríferas apresentam menor requerimento em frio do que gemas vegetativas.

Segundo Monet \& Bastard (1971), existe a possibilidade de necrose de gemas floríferas quando expostas a temperaturas altas. Armas-Reyes et al. (2006) comentam que altas temperaturas $\left(18-20^{\circ} \mathrm{C}\right)$ durante o período de inverno podem interromper o processo de diferenciação das anteras, causando danos aos tecidos meristemáticos e determinando alta queda de gemas florais em Prunus armeniaca L. A exposição contínua à temperatura de $25^{\circ} \mathrm{C}$ após a submissão aos tratamentos de frio pode ter ocasionado danos aos tecidos das gemas floríferas, acarretando reduzida brotação dessas gemas. A ocorrência de acentuada queda de gemas floríferas ao longo do período de avaliação reforça a hipótese de necrose deste tipo de gema pela exposição contínua a $25^{\circ} \mathrm{C}$ após os tratamentos de frio. Frente a esta observação, sugere-se a realização do método biológico com a cultura do pessegueiro, utilizando temperaturas inferiores a $25^{\circ} \mathrm{C}$ após a submissão dos tratamentos de frio.

Em razão dos problemas relacionados à brotação de gemas floríferas encontrados durante a condução deste trabalho, sugere-se a realização de mais estudos para verificar a influência da exposição a baixas temperaturas e da poda de desponte sobre a capacidade de brotação de gemas floríferas em diferentes cultivares de pessegueiro.

TABELA 1- Análise de variância para a variável brotação de gemas vegetativas (GV15 e GV30) de ramos de pessegueiro, das cultivares Eragil, Coral e Rubidoux, após 15 e 30 dias a 25ㄷ․ Caçador-SC, 2006.

\begin{tabular}{lccc}
\hline \multirow{2}{*}{ Fonte de Variação } & \multirow{2}{*}{ GL } & \multicolumn{2}{c}{ QM } \\
\cline { 3 - 4 } & & BGV 15 & BGV 30 \\
\hline Exposição ao frio (EF) & 3 & $145,66^{*}$ & $44,38^{*}$ \\
Cultivar (C) & 2 & $39,02^{*}$ & $41,01^{*}$ \\
Desponte (D) & 1 & $44,72^{*}$ & $79,34^{*}$ \\
EF x C & 6 & $3,49^{*}$ & $1,37^{\text {ns }}$ \\
EF x D & 3 & $17,84^{*}$ & $8,48^{*}$ \\
C x D & 2 & $1,36^{\text {ns }}$ & $0,04^{\text {ns }}$ \\
EF x C x D & 6 & $0,65^{\text {ns }}$ & $1,12^{\text {ns }}$ \\
\hline Erro & 96 & 1,03 & 0,85 \\
CV $(\%)$ & - & 27,67 & 17,82 \\
\hline
\end{tabular}

GL - graus de liberdade; QM - quadrado médio; CV - coeficiente de variação; GV15, GV30 - percentagem de gemas vegetativas brotadas aos 15 e 30 dias a $25^{\circ} \mathrm{C}$, respectivamente; ns - não significativo; * significativo a $1 \%$ de probabilidade de erro; ${ }^{1}$ variáveis transformadas pela equação $\sqrt{(x+1)}$. 
TABELA 2 - Percentagem de brotação de gemas vegetativas de pessegueiro, nas cultivares Coral, Eragil e Rubidoux, após 15 e 30 dias a $25^{\circ} \mathrm{C}$, em ramos com e sem desponte. Caçador-SC, 2006.

\begin{tabular}{|c|c|c|c|}
\hline \multicolumn{4}{|c|}{ Gemas vegetativas brotadas $(\%)-15$ dias a $25^{\circ} \mathrm{C}$} \\
\hline Desponte & Coral & Eragil & Rubidoux \\
\hline Ramos com desponte & A 37,08 a & A $15,44 \mathrm{~b}$ & A 24,67 b \\
\hline Ramos sem desponte & B $19,02 \mathrm{a}$ & $\mathrm{B} 5,66 \mathrm{~b}$ & B 9,72 b \\
\hline \multicolumn{4}{|c|}{ Gemas vegetativas brotadas $(\%)-30$ dias a $25^{\circ} \mathrm{C}$} \\
\hline Desponte & Coral & Eragil & Rubidoux \\
\hline Ramos com desponte & A 47,15 a & A $25,91 \mathrm{~b}$ & A 42,67 a \\
\hline Ramos sem desponte & B $26,56 \mathrm{a}$ & B 9,98 b & B $22,57 \mathrm{a}$ \\
\hline
\end{tabular}

Médias seguidas de letras maiúsculas, distintas na coluna, e letras minúsculas, distintas na linha, diferem significativamente, pelo teste de Tukey, a 5\% de probabilidade de erro.

TABELA 3 - Brotação de gemas vegetativas em ramos de pessegueiro, das cultivares Coral, Eragil e Rubidoux, submetidos a diferentes períodos de exposição ao frio, após 15 e 30 dias a $25^{\circ} \mathrm{C}$. Caçador-SC, 2006.

\begin{tabular}{|c|c|c|c|c|}
\hline \multicolumn{5}{|c|}{ Gemas vegetativas brotadas $(\%)-15$ dias a $25^{\circ} \mathrm{C}$} \\
\hline Cultivar & $0 \mathrm{UF}^{1}$ & $312 \mathrm{UF}$ & $624 \mathrm{UF}$ & 1248UF \\
\hline Coral & $7,04 \mathrm{a}$ & $11,65 \mathrm{a}$ & $43,22 \mathrm{a}$ & $50,28 \mathrm{a}$ \\
\hline Eragil & $1,27 \mathrm{ab}$ & $1,74 \mathrm{~b}$ & $17,83 \mathrm{~b}$ & $21,35 \mathrm{c}$ \\
\hline Rubidoux & $0,00 \mathrm{~b}$ & $0,42 \mathrm{~b}$ & $34,12 \mathrm{a}$ & $34,24 \mathrm{~b}$ \\
\hline \multicolumn{5}{|c|}{ Gemas vegetativas brotadas $(\%)-30$ dias a $25^{\circ} \mathrm{C}$} \\
\hline Cultivar & OUF & $312 \mathrm{UF}$ & $624 \mathrm{UF}$ & 1248UF \\
\hline Coral & $35,80 \mathrm{a}$ & $18,09 \mathrm{a}$ & $44,26 \mathrm{a}$ & $52,26 \mathrm{a}$ \\
\hline Eragil & $21,79 \mathrm{~b}$ & $6,87 \mathrm{~b}$ & $19,98 \mathrm{~b}$ & $29,91 \mathrm{~b}$ \\
\hline Rubidoux & $15,02 \mathrm{~b}$ & $16,20 \mathrm{a}$ & $40,58 \mathrm{a}$ & $51,89 \mathrm{a}$ \\
\hline
\end{tabular}

${ }^{1}$ UF - unidades de frio complementar, segundo modelo de Utah (Richardson et al., 1974); Médias seguidas por letras minúsculas distintas na coluna diferem significativamente, pelo teste de Tukey, a 5\% de probabilidade de erro.
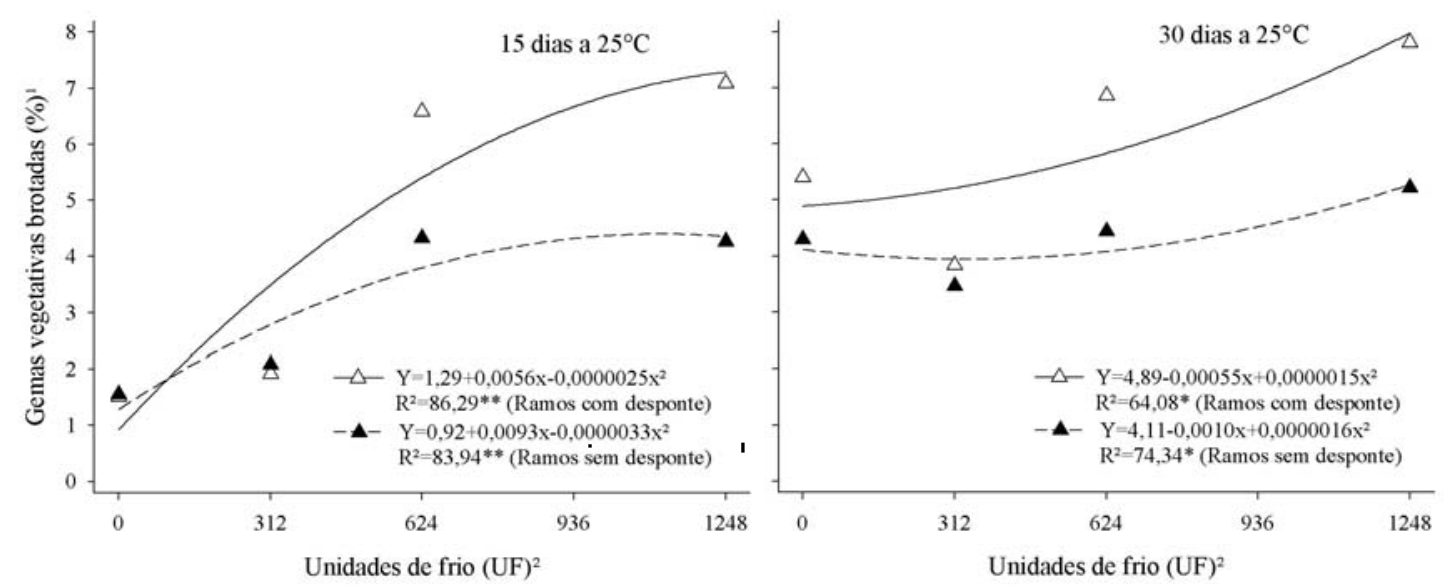

${ }^{1}$ Variável transformada pela equação $\sqrt{(x+1)}$;

${ }^{2}$ Unidades de frio complementar, segundo modelo de Utah (Richardson et al., 1974);

*,** regressão polinomial significativa a 5 e a $1 \%$ de probabilidade de erro pelo teste $\mathrm{F}$, respectivamente.

FIGURA 1 - Efeito da exposição ao frio na brotação de gemas vegetativas em ramos de pessegueiro, despontados e não despontados, após 15 e 30 dias a 25ํ․ Caçador-SC, 2006. 

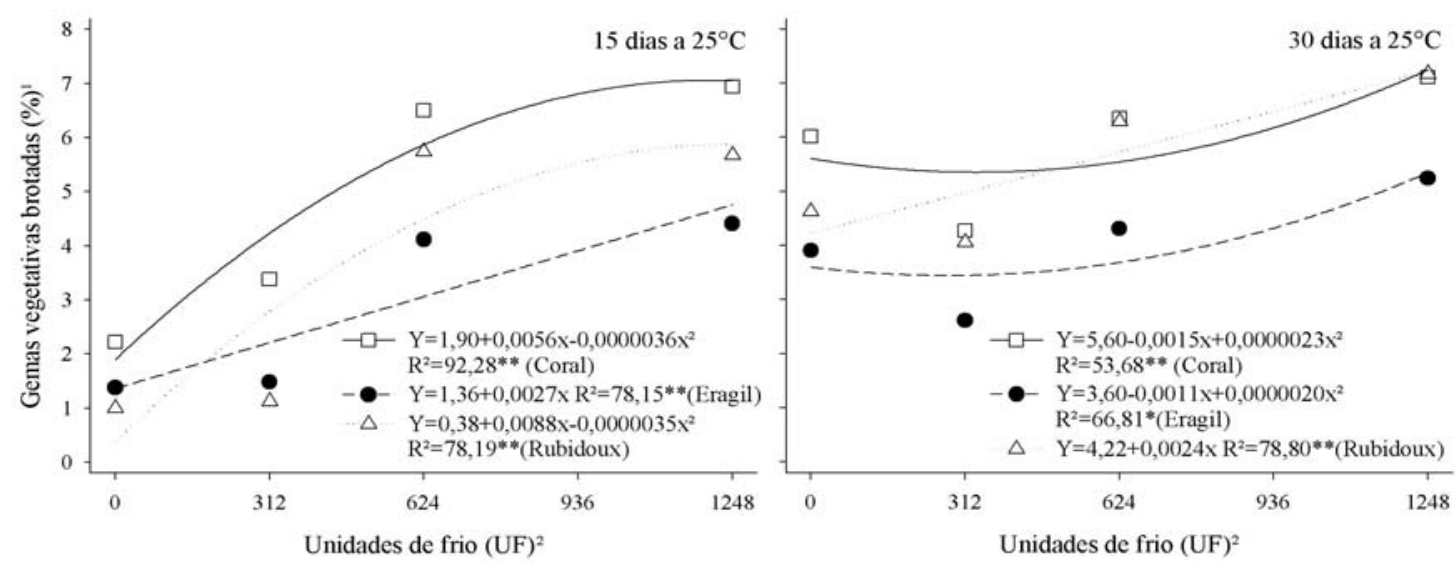

'Variável transformada pela equação $\sqrt{(x+1)}$;

${ }^{2}$ Unidades de frio complementar, segundo modelo de Utah (Richardson et al., 1974);

*,** regressão polinomial significativa a 5 e a $1 \%$ de probabilidade de erro pelo teste $\mathrm{F}$, respectivamente.

FIGURA 2 - Efeito da exposição ao frio na brotação de gemas vegetativas em ramos de pessegueiro, das cultivares Coral, Eragil e Rubidoux, após 15 e 30 dias a 25² C. Caçador-SC, 2006.

\section{CONCLUSÕES}

1-O desponte incrementou a brotação de gemas vegetativas nas cultivares Coral, Rubidoux e Eragil.

2-O efeito do desponte sobre a brotação de gemas vegetativas foi variável conforme o período a que os ramos foram expostos ao frio, sendo mais pronunciado nas exposições superiores a 104 unidades de frio.

3-A metodologia empregada não proporcionou a brotação de gemas floríferas, possivelmente associado à necrose de gemas floríferas pela exposição contínua à temperatura de $25^{\circ} \mathrm{C}$ após os tratamentos de frio.

\section{REFERÊNCIAS}

ARMAS-REYES, R.; CÁRDENAS-SOLORIO, E.; RODRÍGUEZ-ALCÁZAR, J. Conéxion vascular y otros factores que influyen en la caída de yemas florales de chacabano. Revista Chapingo, Chapingo, v.12, n.1, p.33-39, 2006.

BANZATTO, D.A.; KRONKA, S.N. Experimentação agrícola. 3.ed. Jaboticabal: FUNEP, 1995. p. 54-59.

BARRIT, B.H. Sistemas de Conducción Y Poda em Manzano. Posicionamiento de las Ramas Principales. In: CURSO INTERNACIONAL DE FRUTICULTURA DE CLIMA TEMPLADO-FRIO, 1998, Mendoza. Anais... 10p.
BIANCHI, V.J.; ARRUDA, J.J.P.; CASAGRANDE, J.G.; HERTER, F.G. Estudo da paradormência em pereira por meio do método biológico. Revista Brasileira de Fruticultura, Jaboticabal, v. 22, n. 2, p.294-296, 2000.

BIASI, L.A.; ZANETTE, F.; PETRI, J.L.; MARODIN, G.A.B. Cultivares de fruteiras de caroço. In: MONTEIRO, L.B.; MAY-DE MIO, L.L.; SERRAT, B.M.; MOTTA A.C.; CUQUEL F. L. Fruteiras de caroço: uma visão ecológica. Curitiba: UFPR, 2004. p.5-32.

BOTELHO, R.V.; AYUB, R.A.; MÜLLER, M.M.L. Somatória de horas de frio e de unidades de frio em diferentes regiões do Estado do Paraná. Scientia Agraria, Curitiba, v.7, n.1-2, p.89-96, 2006.

CITADIN, I. Necessidade de calor para antese e brotação em pessegueiro [Prunus persica (L.) Batsch]. 1999. 74 f. Dissertação (Mestrado em Fruticultura de Clima Temperado) - Faculdade de Agronomia Eliseu Maciel, Universidade Federal de Pelotas, Pelotas, 1999.

COOK, N.C.; JACOBS, G. Suboptimal winter chilling impedes development of acrotony in apples trees. HortiScience, Alexandria, v. 34, n. 1, p. 1213-1216, 1999.

EREZ,A. Bud dormancy: Phenomenon, problems and solutions in the tropics and subtropics. In: EREZ, A. (Ed.). Temperate fruit crops in warm climates. 
London: Kluwer Academic Publishers, 2000. p.1748.

FAUST, M.; LIU, D.; WANG, S.Y.; STUTTE, G.W. Involvement of apical dominance in winter dormancy of apple buds. Acta Horticulturae, Wageningen, v. 395, p. 47-56, 1995.

HERTER, F.G.; RAGEAU, R.; BONHOMME, M.; MAUGET, J.C. Determinação do término da dormência e floração para algumas cultivares de macieira: comparação entre métodos biológicos e empíricos. Revista Brasileira de Fruticultura, Jaboticabal, v. 14, n. 1, p. 77-81, 1992.

LANG, G.A. Dormancy: a new universal terminology. HortScience, Alexandria, v.22, n.5, p.817-820, 1987.

LEITE, G.B. Évolution des états des bougeons et de leur hétérogénéité le long du rameau d'un an de pêcher sous différents régimes de temperatures après l'installation de l'endodormance. 2004. $168 \mathrm{f}$. Thèse Doutorado - Univesité Blaise Pascal, Montpellier, 2004.

LEITE, G. B. Evolução da dormência e heterogeneidade da brotação In: ENCONTRO NACIONAL SOBRE FRUTICULTURA DE CLIMA TEMPERADO, 8., 2005. Anais... Caçador: Epagri, 2005. p. 269-275.

MONDIN, L.R. Avaliação de diferentes intensidades de poda invernal de desponte na produtividade e na qualidade de pêssegos cultivares Maciel e Granada. 2005. 53 f. Dissertação (Mestrado em Agronomia Fruticultura de Clima Temperado) - Faculdade de Agronomia Eliseu Maciel, Universidade Federal de Pelotas, Pelotas, 2005.

MONET, R.; BASTARD, Y. Effects d'une température moderement éleveé: $25^{\circ} \mathrm{C}$, sur les bourgeons floraux du pêcher. Phisiologie Végétale, Paris, v.9, n.2, p.209226, 1971.
PETRI, J. L. Dormência da macieira. In: EMPASC. Manual da cultura da macieira. Florianópolis, 1986. cap.7, p.163-201.

PETRI, J.L.; PALLADINI, L.A.; POLA, A.C. Dormência e indução a brotação em macieira. In: EPAGRI. A cultura da macieira. Florianópolis, 2006. p.261-297.

PETRI, J.L.; HERTER, F.G. Dormência e indução a brotação. In: MONTEIRO, L.B.; MAY-DE MIO, L.L.; SERRAT, B.M.; MOTTA A.C.; CUQUEL F. L. Fruteiras de caroço: uma visão ecológica. Curitiba: UFPR, 2004. p.49-57.

PUTTI, G. L.; PETRI, J.L.; MENDEZ, M. E. Efeito da intensidade do frio no tempo e percentagem de gemas brotadas em macieira. Revista Brasileira de Fruticultura, Jaboticabal, v.25, n.2, p.199-202, 2003.

RICHARDSON, E.A.; SEELEY, S.D.; WALKER, D.R. A model for estimating the completion of rest for 'Redhaven' and 'Elberta' peach trees. HortScience, Alexandria, v.1, p.331-332, 1974.

TAIZ, L.; ZEIGER, E. Fisiologia vegetal. 3.ed. Porto Alegre: Artmed, 2004. 719p.

TROMP, J. Dormancy. In: TROMP, J.; WEBESTER, A.D.; WERTHEIM, S.J. Fundamentals of temperature zone tree fruit production. Leiden: Backhuys Publishers, 2005. p.65-73.

ZELLEKE, A.; KLIEWER, W.M. The effects of hydrogen cyanamide on enhancing the time and amount of budbreak in young grape vineyards. American Journal of Enology and Viticulture, Davis, v. 40, p. 47-51, 1989. 